\title{
A possible point of contact between cosmic ray physics and archaeology: muon absorption radiography at the Tharros Phoenician-Roman site
}

\author{
Lorenzo Bonechi ${ }^{1,{ }^{\star}}$, Fabio Ambrosino ${ }^{2,3}$, Luigi Cimmino ${ }^{2,3}$, Raffaello D’Alessandro ${ }^{1,4}$, \\ Nicola Mori ${ }^{1,4}$, Pasquale Noli ${ }^{3}$, Giulio Saracino ${ }^{2,3}$, Paolo Strolin ${ }^{2,3}$, Lorenzo Viliani ${ }^{1,4}$ \\ ${ }^{1}$ Istituto Nazionale di Fisica Nucleare (INFN), Sezione di Firenze, Sesto Fiorentino, Florence, Italy \\ ${ }^{2}$ Università di Napoli Federico II, Dipartimento di Fisica, Naples, Italy \\ ${ }^{3}$ Istituto Nazionale di Fisica Nucleare (INFN), Sezione di Napoli, Naples, Italy \\ ${ }^{4}$ Università di Firenze, Dipartimento di Fisica e Astronomia, Sesto Fiorentino, Florence, Italy
}

Article history
Received July 5, 2016; accepted October 5, 2016.
Subject classification:
Muon radiography, Muon absorption, Archaeology, Tharros necropolis.

\begin{abstract}
Several on-going activities exploiting the potential of the muon-absorption radiography technique are focusing on the study of large volcanic structures located in different geographical regions. Nonetheless, the possible application of this non-destructive surveying method to other fields is also under consideration by different groups. Looking backward to the history of muon radiography, we can learn how the first important success of this technique was achieved in the archaeological field by the physicist Luis W. Alvarez in the 1960s. Further examples of possible applications can be found concerning geological and mining applications. In this paper a possible application of muon absorption radiography in the context of the archaeological research is proposed. Results of a simulation of a simplified case study are presented.
\end{abstract}

\section{Introduction}

Many activities exploiting the muon absorption radiography (or muon transmission radiography) technique are currently under consideration or development in fields other than volcanology, as partially reported in other contributions to this workshop. This technique, which can be properly contextualized in the generic framework of monitoring or imaging of material structures, has the potential to be applied in many different areas. Each area of application, and often each application itself, requires an independent feasibility study for this technique to be validated. This is due to several reasons, which can be summarized in the following main points: 1) the different materials and material thicknesses encountered by the incident muons, 2) the muon energy range relevant for the selected case study, 3 ) the relative detector-target geome-try, 4) the type of detector which can be used for the measurement and 5) the purpose of the measurement itself.

The first practical cases where muon absorption radiography was successfully applied were in the fields of geology and archaeology. In the 1950s E.P. George measured the thickness of the ice burden over the GuthegaMunyang tunnel in Australia [Agostinelli et al. 2003].

A decade later L.W. Alvarez studied the interior of the second pyramid of Chephren in Egypt, looking for a hypothetic hidden chamber [Bonechi et al. 2015]. In the latter case, Alvarez was able to demonstrate the absence of any large chamber hidden over the already discovered one.

Drawing on the idea of Alvarez, in this work we propose a possible application of muon absorption radiography in the framework of Archaeology, based on the use of a robust, low cost and low power consumption particle detector. In Section 2 a description of the case study is presented; in Section 3 the preliminary results of a simulation are shortly discussed; finally, in Section 4 the first activities for the production of a prototype detector are presented.

\section{Detecting hidden tombs at Tharros}

The Tharros site (see Figure 1) is a beautiful PunicRoman site located at the south end of the Sinis Peninsula near the town of Oristano in the Sardinia Island (Italy). In 2014 we had contacts with the managers of the site who illustrated some relevant details about the an- 


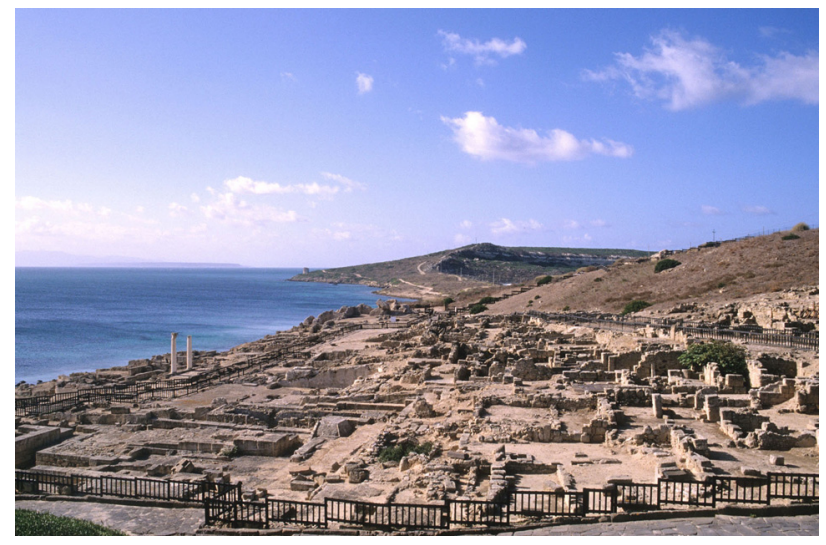

Figure 1. View of the Tharros remains.

cient town. In summer, we had the possibility to visit the site to evaluate a possible usefulness of exploiting muon radiography for a survey of unknown parts of the site.

Apart from the main ruins of Tharros, related to the old town itself, a large area of the peninsula hosts an important necropolis, which is still hidden underground for the greatest part. Only a few points have been brought to light by excavations and later covered again to preserve the status of the site. Part of these excavations are still visible (see Figure 2). Surveys by means of a GPR device have been only partially conducted due to the difficulty of moving this kind of device over large areas covered by the Mediterranean scrub, which is protected by the Italian legislation. Therefore, the presence of tombs in several parts of the site (e.g. Figure 3) is currently only a guess.

Thanks to the muon radiography technique, the placement of a muon detector in a fixed measuring position, possibly ten or fifteen metres underground or under a cliff, would give the possibility to make a survey of some hundred square metre surface searching for empty cavities. The minimum volume of a hidden tomb that can be reasonably detected with this method varies from one to a few cubic metres, assuming the

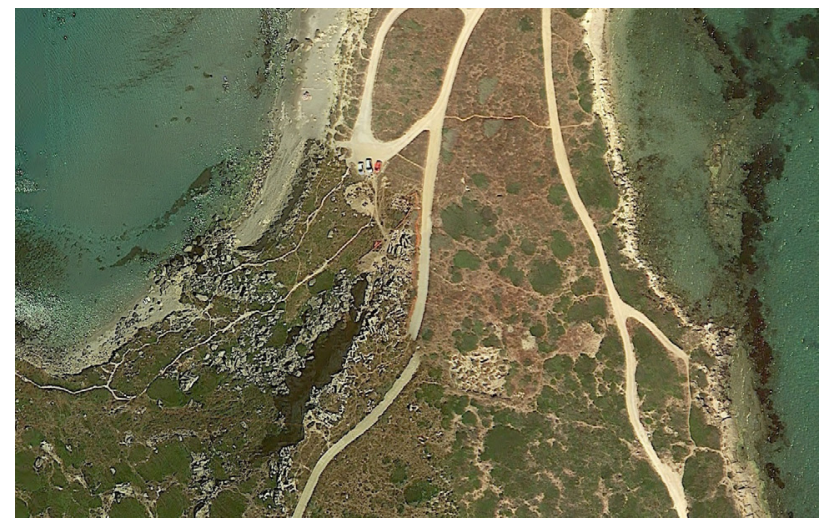

Figure 3. The region of the excavation shown in Figure 2. A necropolis is thought to occupy large parts of this place.

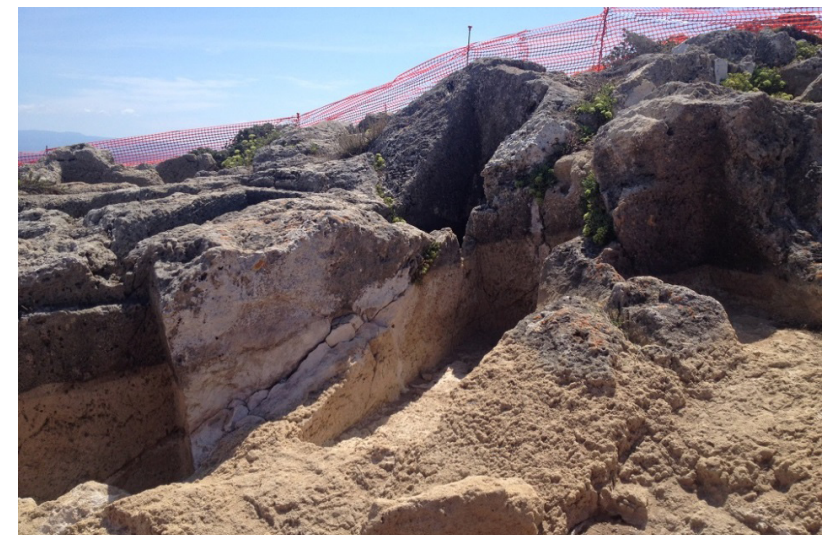

Figure 2. Tombs found in the Tharros necropolis.

tomb is located a few metres underground. To assess the possibility of identifying such hypothetic hidden tombs by exploiting the information carried by the detected muons, a data analysis method [Anastasio et al. 2013] has been proposed, based on the comparison of the measured flux with a simulated expected flux, calculated for the same data acquisition time.

The required simulations must take into account all the relevant aspects of the problem, from the functional shape of the muon energy/angular spectra at ground level, to its modification while traversing the material along the detector's field of view. This method is based on the back-projection of the detected and simulated muon tracks to parallel planes at increasing distances from the detector, or to spherical surfaces centred on the detector's centre for increasing radius. Two hit map distributions are therefore produced for each surface and then subtracted from each other. As a result of the different muon absorption in the real and in the simulated cases, the resulting "difference map" should present regions with an accumulation or deficit of hits beyond the statistical fluctuations in correspondence of a sufficiently large unknown empty cavity or a place containing a material much heavier than the surrounding earth or rock.

\section{Preliminary study with the Geant 4 high energy physics simulation package}

Preliminary simulations of a simplified case study have been implemented based on the Geant 4 simulation package [Agostinelli et al. 2003], a toolkit that is commonly used in high energy physics for simulating the passage of particles through matter. Details of this study are published in Bonechi et al. [2015]. A homogeneous terrain with a flat surface has been considered, with a generic "reasonable" chemical composition. A realistic muon detector, based on the technology used for the MURAY experiment [Anastasio et al. 2013], has been positioned $7 \mathrm{~m}$ underground and a one cubic metre empty cavity has been placed $4 \mathrm{~m}$ undersoil on its field 
of view, $8 \mathrm{~m}$ far from it. A sketch of the com-plete geometry implemented in Geant4 is shown in Figure 4.

A realistic muon flux generator has been integrated in the Geant 4 code. This generator is based on experimental measurements of cosmic rays at ground level by the ADAMO experiment [Bonechi et al. 2005] in the momentum range between $100 \mathrm{MeV} / c$ and 130 $\mathrm{GeV} / c$, which is relevant for this application.

Two different simulated data sets, with and without the presence of the empty cavity, have been generated and the results compared (see Bonechi et al. [2015] for details). For each of these simulations a number of muon events, corresponding to a data acquisition time ranging from two weeks up to 130 days, entering the detector's field of view and passing through a region surrounding the cavity has been generated. Muon tracks are reconstructed and the final trajectories saved to file only if crossing all tracking planes. The sets of reconstructed tracks are back projected to vertical planes or spherical surfaces and a two dimensional hit density map is produced for both simulations in correspondence of each surface, as shortly described at the end of the previous section.

From the comparison of the two simulations, a hit map difference is obtained for all the selected surfaces. Due to geometrical reasons, a focused image of the cavity is expected as the back projection surface approaches the position of the cavity. In Figures 5, 6 and 7 the difference maps on spherical surfaces of radii $1 \mathrm{~m}$, $4 \mathrm{~m}$ and $7 \mathrm{~m}$ are shown in the azimuth-zenith plane.

These figures show how the image of the cavity gets more detailed while the radius of the back projection sphere increase from $1 \mathrm{~m}$ to $7 \mathrm{~m}$ (see the RMS values of the distributions).

As shown in Bonechi et al. [2015], the best focusing of the image of the cavity can be obtained when the back projection sphere (or plane) crosses a region in proximity of the real position of the cavity itself. In Figure 8 the dependence of the width of the reconstructed image along the azimuth axis, as function of the radius of the back projection sphere (equivalent to the distance from the centre of the detector) is shown.

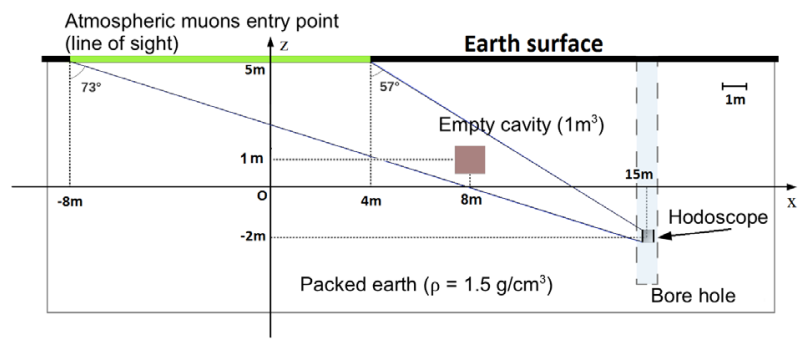

Figure 4. A sketch of the geometry implemented in the GEANT4 simulation.

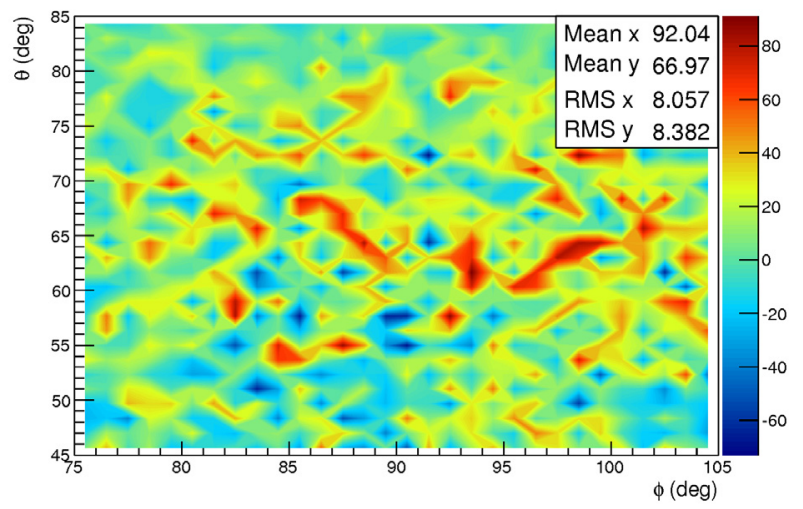

Figure 5. Projection on a $1 \mathrm{~m}$ radius spherical surface.

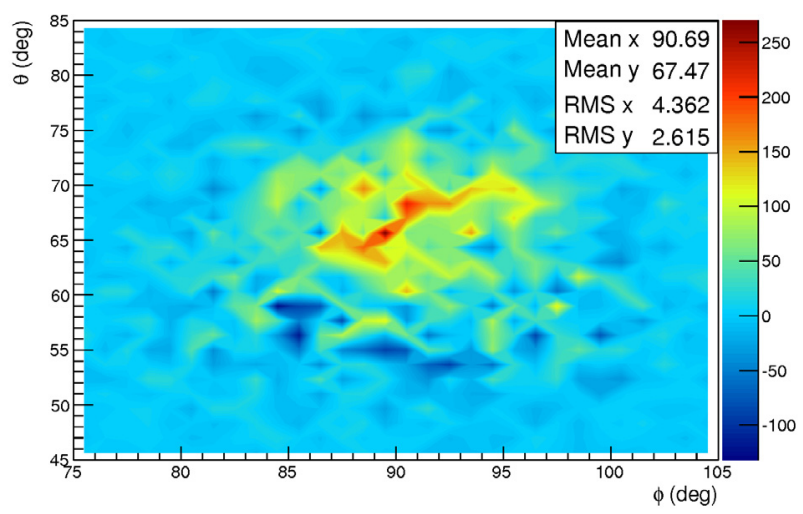

Figure 6. Projection on a $4 \mathrm{~m}$ radius spherical surface.

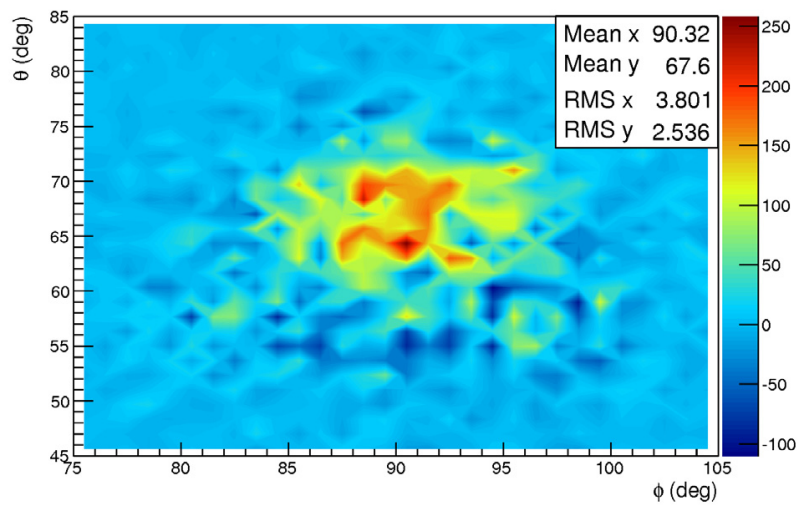

Figure 7. Projection on a $7 \mathrm{~m}$ radius spherical sur-face.

The minimum of this function is found for a distance $d=6.1 \pm 0.7 \mathrm{~m}$, approximately compatible with the position of the face of the cavity closer to the detector. The angle subtended in this example by the cavity with respect to the detector's centre is approximately 125 mrad. A detector with an angular resolution of $10 \div 50$ mrad is therefore required for this measurement.

\section{Development of a prototype borehole tracking detector}

Based on the experience derived from the MURAY experiment, a prototype borehole muon tracker is under development for a demonstration of the technique pro- 


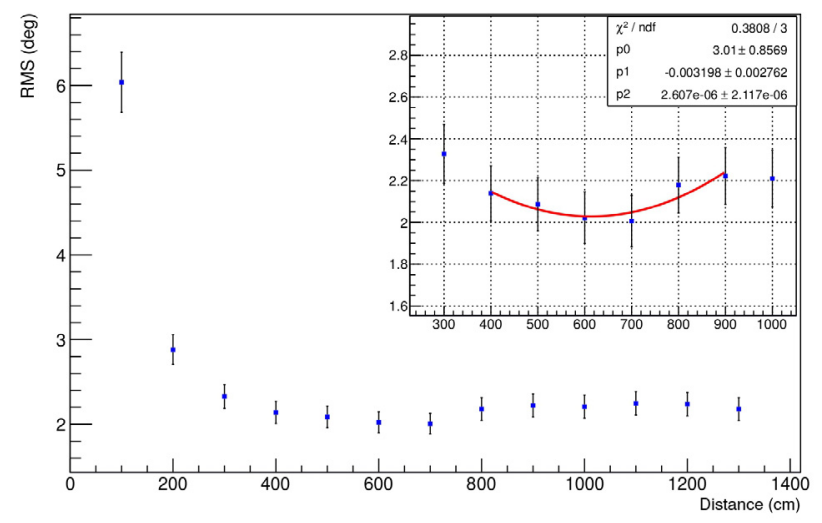

Figure 8. Dependence of the RMS value associ-ated to the image of the cavity as a function of the radius of the back projection sphere. A minimum can be identified for a radius around 6-7 $\mathrm{m}$.

posed in Bonechi et al. [2015]. This detector is a $40 \mathrm{~cm} \times$ $40 \mathrm{~cm} \times 40 \mathrm{~cm}$ cubic tracker made of two $x$ and two $y$ planes. Each plane is made of 22 scintillator bars with isosceles triangular section (Figure 9).

The scintillator material is UPS-923A by De-tecRad, which has a good light yield and a fast rising time.

The bar section has a $40 \mathrm{~mm}$ base and $20 \mathrm{~mm}$ height and the total length of each bar is $40 \mathrm{~cm}$. Each bar is enveloped in an aluminized mylar film to guarantee the optical insulation of the scintillator from the external environment (Figure 10).

The triangular bars are assembled one with the base facing upwards and the adjacent one with the base facing downwards, in such a way to fill a $20 \mathrm{~mm}$ height parallelepiped volume (with oblique faces at two ends).

Signals from the two adjacent bars crossed by a muon can be used to determine the crossing point of the muon tracks with a better resolution with respect to the simple digital algorithm.

For the single tracking plane, made of 22 bars, we expect a spatial resolution around $3 \div 4 \mathrm{~mm}$, according

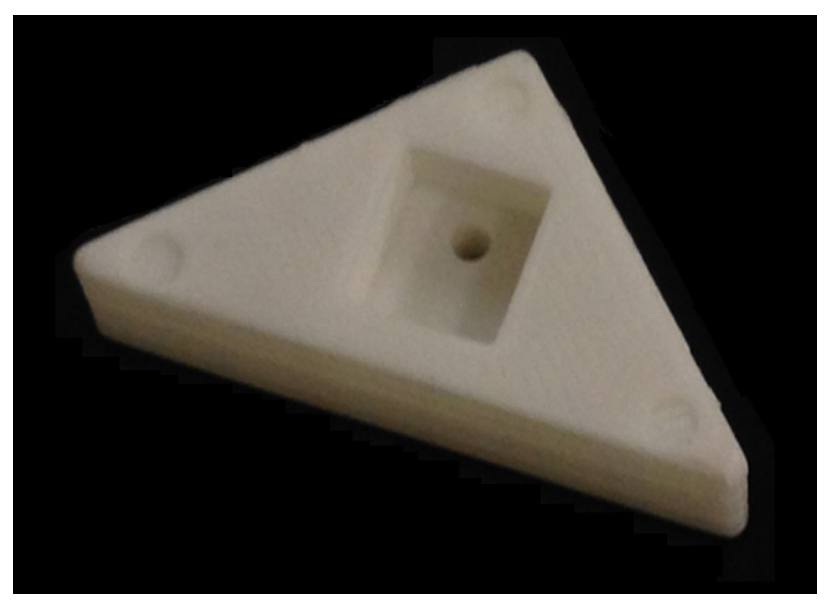

Figure 11. Plastic support use to fix the SiPM sensors to the scintillator bars. At the centre a slot used to host the sensor is visible, with a small hole to drive the signal to the readout electronics.

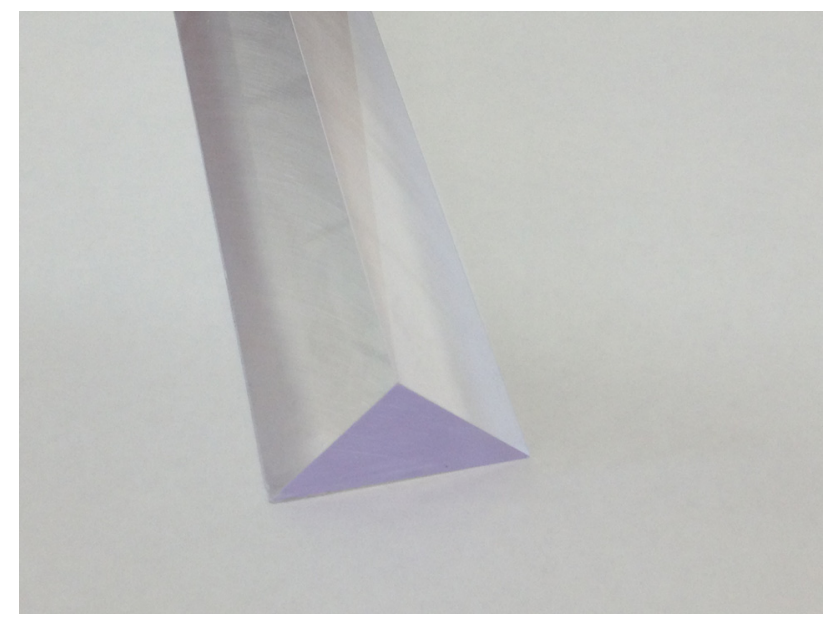

Figure 9. One scintillator bar used for the con-struction of the prototype muon tracker.

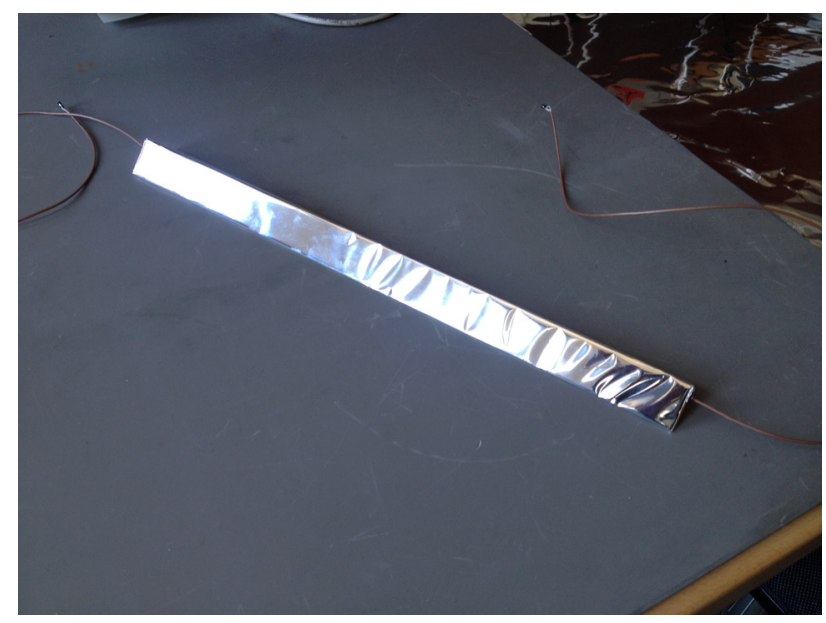

Figure 10. One scintillator bar enveloped in the aluminized mylar film.

to previous tests with similar bars [Carlà 2009/2010]. Considering two planes at $40 \mathrm{~cm}$ distance, it means therefore an angular resolution of $10 \mathrm{mrad}$ or better.

Each bar is readout by two silicon photomultiplier (SiPM), one on each side. The selected model for this device is ASD-NUV4S-P by AdvanSiD, which is a $4 \times 4 \mathrm{~mm}^{2}$ device optimized for near ultra violet light detection. A transparent epoxy layer protects the surface of each SiPM, therefore the optical contact between SiPM and scintillator bar can be achieved by means of a thin layer of optical grease directly applied to the surface of the SiPM. These sensors are kept faced to the triangular faces of the bars thorough dedicated plastic supports produced at a 3D printer. A photo of such a plastic support is shown in Figure 11. These parts are fixed head on to the bars thanks to standard double sided tape. Some pieces of kapton tape is finally applied for safety to fix the support laterally.

The readout electronics is currently under development. It is based on the EASIROC chip by Omega, a 32 channels chip dedicated to readout SiPM detectors. 
The same chip is used for the MURAY and MURAVES projects.

\section{Conclusions}

The possibility to exploit the potential of muon absorption radiography for surveys in the archaeological field is under consideration. In particular, the application of this technique for the study of the necropolis of the Tharros site in the Sardinia island is under consideration. For this purpose a prototype borehole muon tracker based on scintillators and Silicon Photomultipliers in under development. The readout electronics will be similar the MURAY and MURAVES projects. The first test of the technique is foreseen for 2016 in a controlled underground environment at the INFN Unit of Florence (Italy).

Acknowledgements. We would like to thank prof. Anna Chiara Fariselli, who has the ministerial concession for archaeological research at Tharros, dr. Federica Boschi from University of Bologna and dr. Roberto Carrus, president of Cooperativa "Penisola del Sinis", for their availability for discussions and for the invitation to visit the site of Tharros. We would like also to thank Mr. Massimo Falorsi, head of the workshop of the Department of Physics and Astronomy of University of Florence, and Mr. Marco Manetti of INFN Unit of Florence for the production of scintillators and mechanics.

\section{References}

Agostinelli, S., et al. (2003). Geant4 - a simulation toolkit, Nucl. Instrum. Meth. A, 506, 250-303.

Anastasio, A., et al. (2013). The MURAY experiment. An application of SiPM technology to the understanding of volcanic phenomena, Nucl. Instrum. Meth. A, 718, 134-137.

Bonechi, L., M. Bongi, D. Fedele, M. Grandi, S. Ricciarini and E. Vannuccini (2005). Development of the ADAMO detector: test with cosmic rays at different zenith angles, Proceedings of the 29th International Cosmic Ray Conference, 9, 283-286.

Bonechi, L., R. D’Alessandro, N. Mori and L. Viliani (2015). A projective reconstruction method of underground or hidden structures using atmospheric muon absorption data, J. Instrum., 10, P02003.

Carlà, M. (2009/2010). Misura della risoluzione spaziale di un odoscopio realizzato con scintillatori a sezione triangolare, Bachelor's Degree in Physics, Academic Year 2009/2010, University of Florence, Italy (in Italian).

${ }^{\star}$ Corresponding author: Lorenzo Bonechi,

Istituto Nazionale di Fisica Nucleare (INFN), Sezione di Firenze,

Sesto Fiorentino, Florence, Italy; email: lorenzo.bonechi@fi.infn.it. 